\title{
Erratum
}

\section{Erratum to "Study of the Nonlinear Dropping Shock Response of Expanded Foam Packaging System"}

\author{
Huan-xin Jiang, ${ }^{1}$ Yong $\mathrm{Zhu}^{2}{ }^{2}$ and Li-xin $\mathrm{Lu}^{1}$ \\ ${ }^{1}$ Jiangsu Province Key Laboratory of Advanced Food Manufacturing Equipment and Technology, Jiangnan University, \\ Wuxi 214122, China \\ ${ }^{2}$ Key Laboratory of Product Packaging and Logistics of Guangdong Higher Education Institutes, Packaging Engineering Institute, \\ Jinan University, Zhuhai 519070, China \\ Correspondence should be addressed to Yong Zhu; zymmpi@126.com \\ Received 15 September 2013; Accepted 23 September 2013 \\ Copyright ( 2013 Huan-xin Jiang et al. This is an open access article distributed under the Creative Commons Attribution License, \\ which permits unrestricted use, distribution, and reproduction in any medium, provided the original work is properly cited.
}

In the paper titled "Study of the Nonlinear Dropping Shock Response of Expanded Foam Packaging System" the affiliations of the authors are corrected here. 


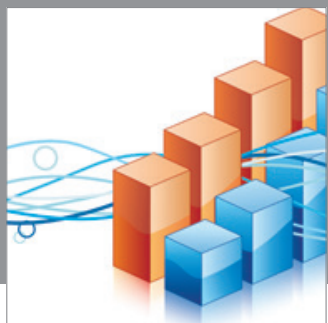

Advances in

Operations Research

mansans

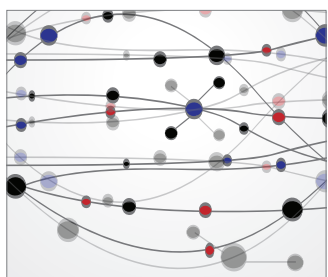

The Scientific World Journal
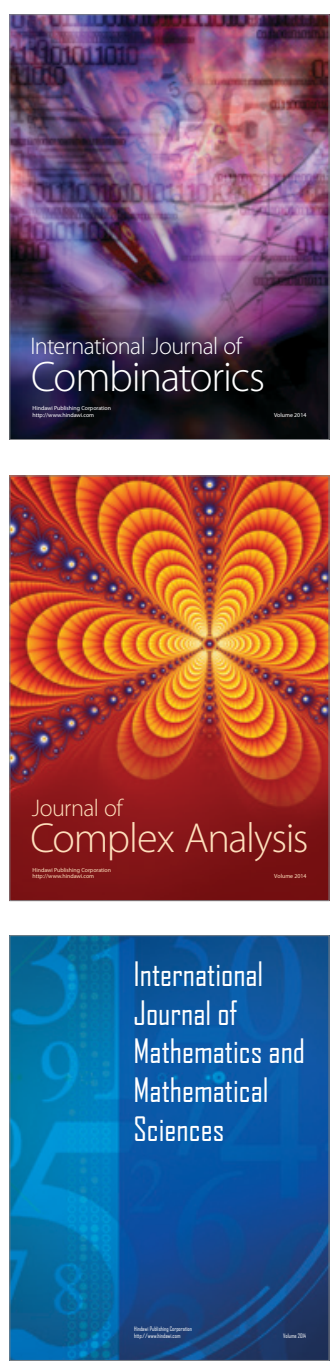
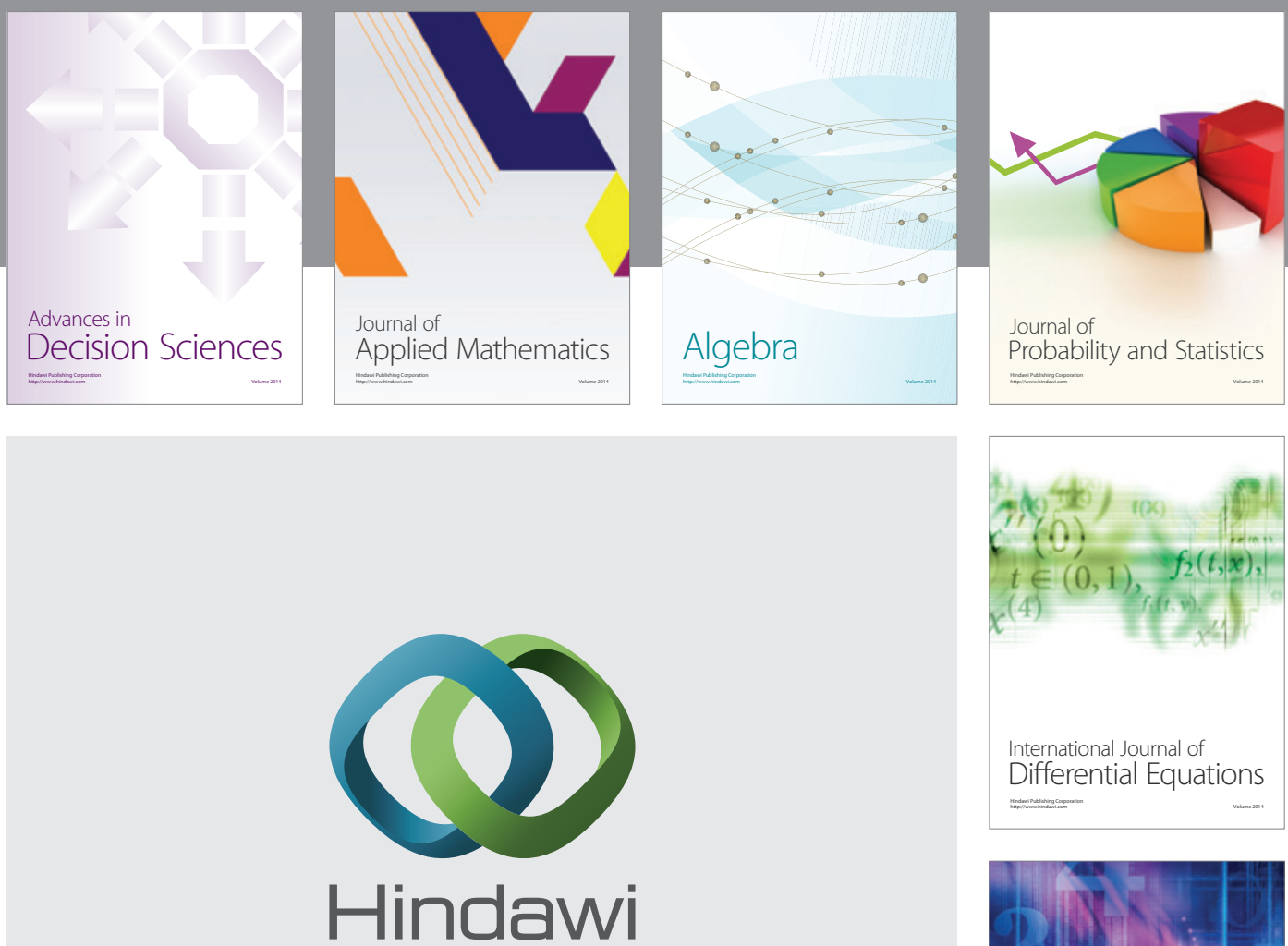

Submit your manuscripts at http://www.hindawi.com
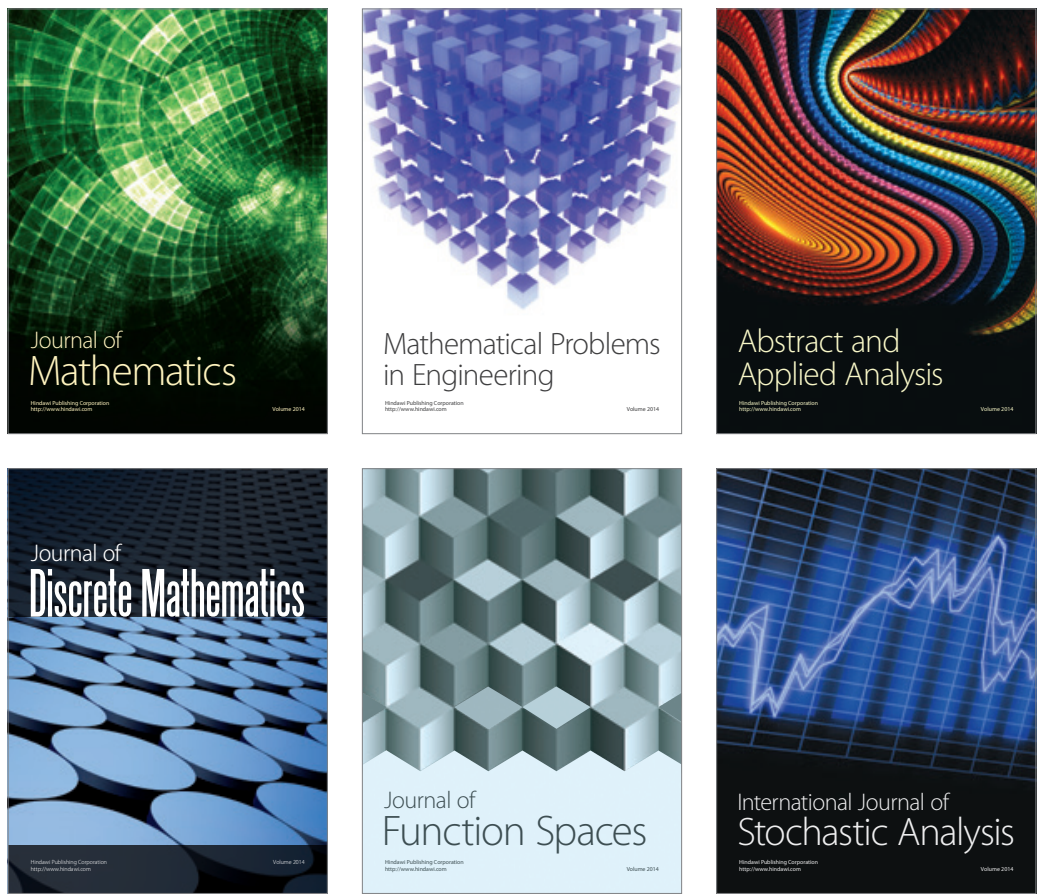

Journal of

Function Spaces

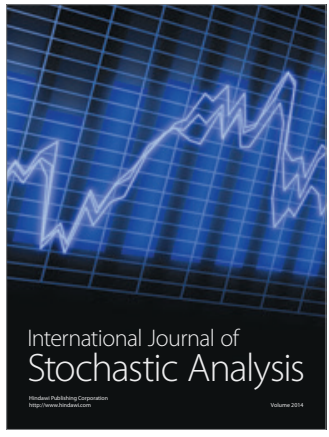

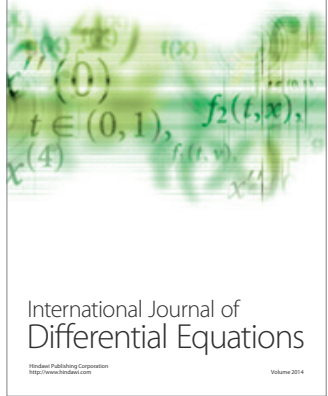
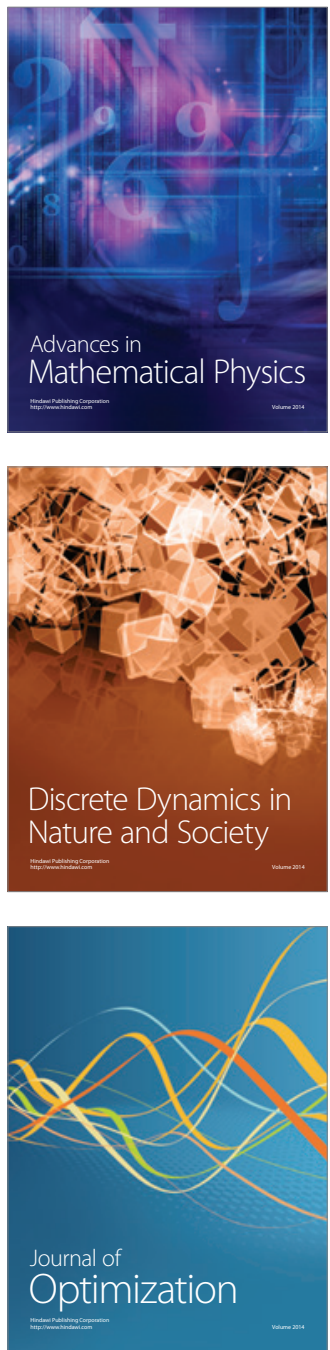